CORRELATION EFHECTS IN PHOTOELECTRON SPECTROMETRY OF RTOMS

$$
\text { David-A: Shirley }
$$

Materials and kolecular Research Divișion Lawrence Berkeley Laboratory and

Department of Chemistry university. of California Berkeley, California 94720 U.S.A.

ABSTRACT

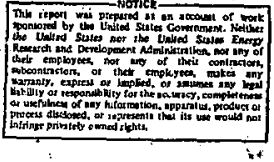

As intense, continuousiy-tunable photon sources become available throughout the vacuum-ultraviolet and soft-x-ray energy, range, it is useful to survey the efferts that can be studied using photoelectron spectrometry. Such a survey is presented, and examples are cited to illustrate the current state of our knowledge in this area.

Photoelectron spectrometry differs from absorption studies in that kinetic energy analysis of electrons yields information about each channel in a photoionization event, rather than yielding only the total cross-section. Electron angular distribution measurements can also provide further information about spins and multipolarities. Probably the most intriguing feature of electron spectrometry is the direct irsight that it can give into electron correlation effects. In 2 general description of electron correlation effects there is no necessity to consider one-electron orbitals. . However, as a heuristic device it is convenient to do so, and to consider at least fout " "ferent" manifestations of electron corxelation in electron spectrometry: initial-state configuration interaction (ISCI), finalionic-state configuration interaction (FISCI), continuum-state configuration interaction (CSCI), and autoionization. There now exist 
rather well-undergtoog examples of cr satellites, which will be cited, and reviewed. Future directions that further studies of these and related phenomena might take to better understand electron correlation. In atonies will be discussed:" 


\section{I: Introzuction}

Electron-electron correlation 'plays an important role' in the electronie stxucture and properties of atonis. Any real improvement of our theoretical understanding of many-electron systems, beyond that ifforded by the one-electron nodel, is poisible only, if correlation. is taken into account. - several experimental manifestations of electron correlation have been known in optical spectroscopy for many years. ' Phese include, for example, deviations of line intensittes or energy interval spacin. from expectations based on an independentelectron model. With the advent of tunable-energy synchrotron radiation it has become possible to extend absorption measurements into the vacuun ultraviolet and to study such manifestly correlationdependent effects as autoionization. We may note as a good example of autoionization in the vacuum ultraviolet che absorption spectrum of atomic barium near the $5 p$ ionization threshold, reported by Connerade, et al. 2

With such an abundance of optical data available, collected over the years by many wcrkers, a great deal of information about electron correlation in atoms is already available. We should ask, then, at the outset: what can photoelectron spectrometry tell us about correlation that is new and unique?

The answer may be given in two parts. First, photoelectron spectrometry clearly affords the possibility of obtaining much more information about an atomic system than does an absorption experiment. This point is illustrated in Fig. 1, which shows that photoelectron 
spectrim of atonic barium as excfted by a laboratory ter lamp, together with the barium energy-level scheme. ${ }^{3-5}$ This detalled spectrum was obtained in large measure by the use of a single photon energy (with some contribution from electrons ejected by sateliztes of the main HeIa line). : Each of the autoionization resonarces in the absorption spectrum would presumably yield a similar electron spectrum, differing in detail. clearly a wealth of additional information is provided by electron spactrometry.

Second; the final states reached in photoelectron spectrometry and the intensity with which they are populated - give a very direct indication of the nature of the correlation beisg studied. This is particularly evident in a configuration-interaction framework. It is a consequence of the fact that electrons are being removed singly and the resultant states observed directly.

In this paper we shall review first the observation of finalionic-state correlation (FISCI) by electxon spectrometry, in section II. The main principles are illustrated by selected examples. Initialstate correlation (ISCI) is treated next (Section III). In Section IV several cases are reviewed in which both ISCI and final-state CI play important roles. Finally, the relation between batellite structure in $x$-ray emission and $x$-ray phitoelectron spectra is discussed briefly. In section $v$.

\section{Finai-Ionic-state Confiquration Interaction (FISCI)}

Final-state conflguration interaction can be classified in the three categorles of autoionization, a resonant process that actually 
encompasses the other two (treated briefly in section IV), and the ron-resonant processes of final-ionic-state CI (FISCI) and contiruumstate CI (CSCI), which Includes the urbound electron. In this section we discuss FIsCI although CSCI is undoubtedly also present in some or all of the cases clted below.

The oldest. and best-known example of FISCI is the "shake-up" phenomenon, studied particularly in rare gases by Carlson, Krause, and co-workers. ${ }^{6}$ Here, FISCI is manifest through weak satellites at higher binding energies accompanying the Ne 1s, etc. peaks. The. origin of these peaks is shown diagramatically in Fig. 2. 7,8 Time evolves upward in this diagram. The first vertex corresponds to photoemission of a is electron from neon into a continuum $p$ state (Ep). Collapse of the residual valence shell to shield the Is hole thus created is denoted by the second vertex, in, which a $3 p$ electron and a $2 p$ hole are created. This is a useful diagram, but the mechanism must not be taken literally (as in any diagram). The final state a $\mathrm{Ne}^{+}\left(\operatorname{ls} 2 \mathrm{~s}^{2} 2 \mathrm{p}^{5} 3 \mathrm{p}\right)$ state - exists independently of photoemission and is reached by exactly the same mechanism as is the "main" $\mathrm{Ne}^{+}\left(1 \mathrm{~g} 2 \mathrm{~s}^{2} 2 \mathrm{p}^{6}\right.$ ) state. 9 shake-up of this kind, accompanying core ionization, is often given a speciai designation, such as "core rearrangement". 7 we emphasize, however, that it is operationally indistinguishable from other cases of FISCI.

The 35 shell in $\mathrm{Mn}^{3+}$ provides the next example of FISCI. ${ }^{10}$ In this case, as illustrated diagranmatically in Fig. 3, a 3 s electron may be regarded as being photoejected into an EP state. The $3 s$ hole is then converted into a $3 p$ hole, with another $3 p$ electron being 
simultaneously excited Into a 3d onbital. In tho language of configurátion Inţeraction which Bagus, et al 't used to describe this. case originally; the nominal primary final state configuration $3 s 3 p^{6} 3 d^{5}, 5 s$ - is essentially degenerate with $3 s^{2} 3 p^{4} 3 d^{6}, 5$ s (which forms two levels). The latter configuration can be formed fron the former by the promotion $\mathrm{p}^{2} \rightarrow \mathrm{gd}$. The energy cost is very slight because the a orbital lies below the $\dot{p}$ orbital." Conflguration interaction among the three above levels leads to three ${ }^{5} \mathbf{s}$ peaks in the photoelection spectrum, as observed. ${ }^{\prime}$ This case is sightly complicated by multiplet splitting, which gives rise to a ${ }^{7} \mathbf{s}$ state as tiell, jut that state can be ignored for the present discussion. Incidentally; a single ${ }^{7}$ peak and several ${ }^{5} \mathrm{P}$ peaks are also observed in the $\mathrm{Mn}^{3+}$ spectrum, ${ }^{12}$ but their origin is multiplet coupling within the $\mathrm{p}^{5} \mathrm{~d}^{5}$ configuration. ${ }^{13}$ The diagram' in Fig. 3 also applies as writtien to the well-known case of satellite structure in the $3 \mathrm{~s}$ photoelection spectrum of argon. The configuration is $1 \mathrm{~s}^{2} 2 \mathrm{~s}^{2} 2 \mathrm{p}^{6} 3 \mathrm{~s} 3 \mathrm{p}^{6}$ in this case, and the $\mathrm{p}^{2}+\mathrm{sd}$ transfer adruixes comfigurations such as $1 s^{2} 2 s^{2} 2 p^{6} 3 s^{2} 3 p^{4} 3 d$. The first slue to this behavior was provided as $E=r l y$ as 1965 , when Bagus ${ }^{14}$ found that $\triangle S C F "$ calculations gave an $A r(3 s)$ binding energy about 4 eV above the experimental value., More recently, Luyken, et al. ${ }^{15}$ surmised that admixture of the configuration $3 s^{1} 3 p^{6}\left({ }^{2} s\right)$ and $3 s^{2} 3 p{ }^{4}$ nd $\left(^{2} s\right)$ was necessary to explain the experimental intensities in the $\mathrm{Ar}^{+}$optical 3pectrum. Spears, et al. ${ }^{16}$ found intense satellite structure in the 2x 3s,3p photoelectron spectrum, as listed in Table I. They Interpreted the'strongest satellite peaks, at binding energies $9.4 \mathrm{eV}$ and $11.9 \mathrm{ev}$ above the $3 \mathrm{~s}$ peak as arising in this way. A less interse 
shoulder aț 8.1 ev was attributed țo a ${ }^{2}$ p final state in $\mathrm{Ar}^{+}$, which would be a satellite of the $3 p$ hile state. To test these assignmenta, and to understand the $\mathrm{L}_{2,3} \mathrm{x}$-ray emission satellites rejorted by cooper. and Lavilla, ${ }^{17}$ R. L. Martin in our laboratory carried out a comprehensive configuration interaction calculation ${ }^{18}$ on $\mathrm{Ax}^{+}$. We summarize these resuita below, inasmuch as this calculation represents a serious attempt to understand the satellite structure quantitatively.

Martin used a set of slater-type orbitals (STO) optimized by Bagus, ${ }^{14}$ to which he added optimized $3 d, 4 p$, and $5 p$ sTO's. A number of Rydberg orbitals were also included as virtual orbitals, as well as additional $3 \mathrm{~s}$ and $\exists_{p}$ SIO's which proviọed for polarization in the $\mathrm{n}=3$ shell. Finally eight reference configurations were selected and a configuration list was developed which conslsted of the union of all single replacements of orbitals in the reference list with virtual orbitals. A total of 300 configurations were thus included in the CI calculation.

The ${ }^{2} \mathrm{~F}$ manifold based on the $3 p$ hole state was found to be unimportant. The strongest satellite - of $3 s^{2} 3 p^{4} 4 p$ character - was found to Iie $7.3 \mathrm{eV}$ above the main $3 s$ hole peak, with 1.28 of its intensity. The $3 s^{2} 3 p^{4} 4 s\left(^{2} s\right)$ state was calculated to lie nearby, with 2.58 intensity. Thus the shoulder reported by Spears', et al. appears to be of mixed ${ }^{2}$ and ${ }^{2} S$ origin, as inaicated in Table $I$.

The two main satellite peaks are calculated to arise from $3 s^{2} 3 p^{4} 4 q_{i}^{2} s$ at $10 \mathrm{ev}$ and $3 g^{2} 3 p^{4} 4 d_{i}{ }^{2} s$ at $12 \mathrm{ev}$. The intensity of the Eormer is in good agreement with experiment, while that of the latter 
is much too high. This follops because the $3 s^{2} 3 p^{4} 4 d$ state interacts strongly with the shake-off continuum based on $3 g^{2} 3 p^{4} \in d$, as noted by * Luyken, et al. ${ }^{15}$ and by arusia, et al.19 Martin found (Table I. Basis 2) that inclusion of a $3 d$ STO with $\xi=3.5$ (to. simulate the continuum function), reduced the $12 \mathrm{eV}$ peak: intensity from 258 to 188 , confirming this idea. We note that for this valence-shell case, the x-ray emission satelifite intensities are about equal to those from XPS. Finally we note that inclusion of FISCI increased the probability of "multiple excitation" from $4 \%$ to 40 s while reaucing the $3 \mathrm{~s}$ binding energy by $7 \mathrm{ev}$.

From the ubove two examples, one might infer that FrscI might lead to sate,lites on the $s$ hole peaks of XPS spectra rather generally, because of the $5 p^{6}+s^{2} p^{4} d$ excitation. This is in fact the case. ${ }^{18}$. Table II shows the systenatic variations in such sateliites' energy separations for $3 \mathrm{~s} .49$, and $5 \mathrm{~s}$ shells. The $5 \mathrm{~s}$ shell of $\mathrm{xe}$ provides a very interesting case, discussed in Paper I-27 of this conference. 20 In this case, many-body techniques have been employed to account for the complex origins of satellites.

We turn now briefly. to an extreme case of FIsCI : the $4 p$ spectrum of bariun. Wendin and Ohno have discussed this case in detail. 21 The reader is referred to their paper for a full discussion. He mention the $B(4 p)$ case here because of its importance and because of certain unique features.

: Absorption 22 and photoelectron spectroscopy ${ }^{23}$ studies of the $4 s_{1} p$ region of xenon showed that no $4_{1 / 2}$ state could be identifled, 
and that both the $4 \mathrm{~s}$ and " $4 \mathrm{p}_{3 / 2}$ " states fall at energies lower than expected on the basis of $\Delta S C F$ calculations. In fact the " $4 p_{3 / 2}$ " peak show sd structure and the $4_{1 / 2}$ region showed many weak peaks in the photoelectron spectrum. Wendin and ohno showed that FIscr accounts for all of these effects. In the $4 \mathrm{~s}$ case, the $\mathrm{Xe}^{+} 4 \mathrm{~s}$ unmixed hole state Iles just below the $\mathrm{Xe}^{++} 4 \mathrm{p}^{5} 4 \mathrm{~d}^{9}$ ionization threshold. It mixes with the $4 p^{5} 4 d^{9}$ ef continuum states as well as aiscrete $4 p^{5} 4 a^{9} n f$ Rydberg states below the threshold. Wendin and onno describe this process as "dipole fluctuations" in the language of many-body theory. It has also been termed a "vixtual coster-Kronig" or "virtual supex Coster-Kronig" 24 process, depending on whether the specific channel under discussion was an NNO or NWN transition, respectively (e.g.. $4 p^{5} 4 d^{10} \rightarrow 4 d^{9} 5 p^{5}$ nd or $\varepsilon d$, versus $4 p^{5} 4 d^{10}+4 p^{6} 4 a^{8}$ nf or $\left.\varepsilon f\right)$. In the 4s case the As state is thus substantially admixed, and lowered in energy by 9 ev below the $\triangle S C F$ position, but is still an identifiable 4s state. The $4 p_{1 / 2}$ and $4 p_{3 / 2}$ one-electron states, on the other hand, lie above the onget of the $x e^{2+} 4 d^{8}$ continuum and are admixed much more strongly with conflgurations such as $4 d^{8} n f, 4 d^{8} \mathrm{Ef}$, etc. The $\mathbf{A p}_{1 / 2}$ state is thereby completely oblitexated and adrixed into many small peaks, while the main peak corresponds to the lowest state in the spectrum. It is more accurately characterized as $4 a^{8} 4 \pm$ than as $4 p_{3 / 2}$ (as its structure proves), but is strongly mixed, A contributing diagram is depicted in Fig. 4. In the $\mathrm{Xe}^{+} \overline{4 s}$ case FISCI is.thus very stroig, and in the $\overline{4 p}$ case, the one-electron picture is actually meaningless, as Wendin and ohno have pointed out. 


$$
\therefore-8-
$$

III Initial-state Conflguration Interaction (ISCI)

Photolection spectrá are also strongly affected by electron correlation in the inftial state. This is particulaxly easily understood in the cI framework. For example, the valence shells of the Groups IIf and IIB elements are nominally iss ${ }^{2} s$. However, $n p^{2}, n d^{2}$, $n f^{2}$ configurations can also mix into the ground state (in ${ }^{1} s$ couplingi. Whereas.ns ${ }^{2}$ can yield only nsep final states, the ionic states np, nd, nf, etc., may be reached and observed as satellites via photoemission involving the admixed configurations. A diagrammatic represention is given in Fig. 5.

To date, Isci has been reported in the $\mathrm{ns}^{2}$ cases $\mathrm{2n},{ }^{25} \mathrm{Cd},{ }^{25,26}$ $\mathrm{Hg},{ }^{25,27} \mathrm{Ca}^{25} \mathrm{sx}^{25} \mathrm{Ba},{ }^{25} \mathrm{sm}^{5} \mathrm{Eu},{ }^{5}$ and $\mathrm{rb} .^{5}$ a typical spectrum that of calcium excited by HeI radiation - is r"own in Fig. 6 . The Is t. peaks $4 p, 3 d, 5 s$, and $4 d$ are clearly visible. It would be valuable to interpret the intensities of these peaks quantitatively, perhaps leading to a quantitative evaluation of the ground-state admixtures of $4 \mathrm{p}^{2}, 3 a^{2}$, etc. Unfortunately, this will require more knowledge of photoionization cross-sections than is presently available. configuration-interaction calculations ${ }^{28}$ by $\mathrm{KIm}$ and Bayus and by Hansen have shown $\mathrm{d}^{2}$ admixtures into the $\mathrm{g}^{2}$ ground state of Group II atoms, but with amplitudes too small to explain the observed intensities withodt other factors (i.e., cross-section enhancement or other channels) .

ISCI effects have also been studied in the $s^{2} p^{2}$ case of $\mathrm{Pb}$ vapox 29 and In the $\mathrm{s}^{2} \mathrm{p}^{3}$ case of $\mathrm{Bi}$ vapor. ${ }^{30}$ of special interest is the predomiriatitly $\mathrm{p}_{1 / 2}$ character of the $\mathrm{p}^{2}$ ground state in $\mathrm{Pb}$. This is a 
consequence of spin-oxbit splitting and again well-known in optical spectroscopy. ${ }^{31}$ The photoelectron spectrum of $\mathrm{Pb}$ shows an intense $\mathrm{Pb}^{+2} \mathrm{P}_{1 / 2}$ line and only a very weak ${ }^{2} \mathrm{P}_{3 / 2}$ line. Several ISCI satejlites are present, originating from $s p^{2} d$ and $s^{2} d^{2}$ ajimixtures in the ground state.

\section{Combined ISCI and Final-state Effects}

Strictly speaking, a completely rigorous separation of correlation effects in the initial and final state is usually not possible. This is clear in the framswork of configuration interactions because the description of configurations in terms of one-electron orbitals is always sorewhat arbitrary. It is still convenient (and quite accurate), however, to classify many cases as either ISCI or FISCI; indeed this was the basis of the above sectiong. In some cases initial- and finalstate CI are both present to such an extent that both must be considered to obtain a description of the spectrum that is even approximately correct. Two such cases are discussed here.

The first is autoionization in the $5 p^{6}$ shell accompanying resonant excitation in the light rare earths, 5 together with IscI. Among the types of final interactions, autoionization alone is a resonant process, while ISCI, of course, is not. Starting with barium $(z=56)$, in whlch autoionization by HeI resonant radfation i's well-known (Fig. 1), the $5 p^{5}$ ionization threshold does not increase rapialy with increasing atomic number, because the added $4 f$ electrons in large measure shield the concomitant added ruclear charge. In the heavy rare earth, ytterbium $(z=70)$, there is no evidence for fel-induced auto- 
ionization, indicating that the $5 p^{5}$ threshold is too high. 5 Recent unpublished tov absórpition spectra by J. P. Connerade, et al. confirm this picture. As a result, the light rare earths possess $5 p^{5}$ thresholds that lie within reach of the HeId line or its higher-energy satellites, leading to autoionization. At the same time these elements shouid show ISCI in their valence $s^{2}$ configirations. In fact only or predominantly - ISCI is present in their Neí photoelectron spectra. for which the photon energy is too low to permit autoionization. 5 This result was found in both $5 \mathrm{~m}$ and $\mathrm{Bu}$, both of which also showed sutoionization under irradiation with the HeIa lines. In some cases the same final states could be reached by both paths. For example, the $41^{6} \mathrm{sd}$ states of $\mathrm{sm}^{+}$could be reached directly by ionization of the $4 f^{6} 5 d^{2}$ configuration admixed into the nominally $4 f^{6} 6 s^{2}$ ground state of Sm. It could also be reached through the path

$$
5 p^{6} 4 f^{6} 6 s^{2} \stackrel{h v}{\longrightarrow}-5 p^{5} 5 a 4 f^{6} \cdot s^{2} \stackrel{a \cdot i .}{\longrightarrow} 5 p^{6} 4 f^{6} 5 d+e^{-}
$$

where the two steps denote resonant excitation of a Rydberg state followed by autoionization, with both 6 s electron being lost in the second step; i.e., $6 s^{2}+5$ pEP. Separation of the contributions ane to autoionization and to ISCI would yield a wealth of information about electron correlations in these atoms. It will be a very complicated problem, however, which can be 'greatly aided by the use of monochromatic, tunable synchrotron radiation to separate the resonant and the nonresonant process.

For the second example of combined ISCI and final-state CI 
effects, we return briefly to the case of shake-up in the Ne(1s) photoelectron spectrum. Gelius ${ }^{23}$ reported a high-resolution XPS spectrum in which some 13 sateliites of the main Ne(1s) hole-istate peak were 1dentified. In unpublished final-gtate CI calculations in 1969, P.' Bagus and 0 . Gelius had shown that the energy separations of these sateliftes could be fitted rather well. The intensities remained a problem, however; until kartin ${ }^{9}$ found that ISCI was equally important in determining the transition strengths. to these satellites. Thus a $\mathrm{Ne}^{+}\left(1 \mathrm{sis} 2 \mathrm{p}^{2}{ }^{5} \mathrm{p}\right)$ state can we reached either through FISCI, as previousIY believed, via the admixture of $\mathrm{Ne}^{+}\left(1, \mathrm{~s}^{\prime} \mathrm{s}^{2} \mathrm{2R}^{6}\right)$ into it, ar through ISCI, via phototoniaation of a $1 s^{2} 2 s^{2} 2 p^{5} 3 p$ component in the ground state. In fact it is possible to make a fairly direct estimate of the relative importance of various configurations mixed into the ground state, from visual inspection of the intensities of the shakeup satellites. This means that shake-up spectra are of interest generally, rather than only to photoelectron spectroscopists.

\section{v. Satellite Intensities in XES and XPs}

In section II we noted that the relative intensities of the $\mathrm{Ar}^{+}$ $(\overline{3 s})$ satelifte structure were about the same, relative to the main line, in $x$-ray emission spectra and $x$-ray photoemission. Theory and experiment were in agreement on this point, and it was also noted that the satellites were separated by several $\mathrm{eV}$ from the main peak in both cases. Rather general argurents can be made ${ }^{18}$ to show that this behavior should be observed whenever valence-to-core $x$-ray transitions are studied in conjunction with the relevant valence-shell 


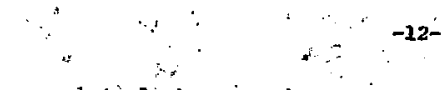

x-ray photóelectron spectra.

of equal interest is the case of core-to-cone $x$-ray enisston spectra; in which the atellite intenstites are usually very small, in contrast to the very intensel sateliltes abserved in xps spectra - of the same (outer) core 1evels. For examie, Asada, et al. 32 "noted that in $\mathrm{N}_{2} \mathrm{~F}_{2}$ a strong_satellite structure appears In Xps on boith che $2 \mathrm{p}_{1 / 2}$ and the $2 \mathrm{p}_{3 / 2}$ 11nes, while the $\mathrm{K} \alpha_{12} \mathrm{k}$-ray lines show no such structure. Martin, et al. 9 have given a simple model that appears to explain this resuit, as shown in Fig. 7 .

Let us approach this problem by considering the $\mathrm{Ni}^{2+}-1$ igand moiety; e.g.. the cluster $\mathrm{NiF}_{6}^{4-}$. This cluster would have a ground state and a spectrum of excited states at optical énergies. If the charge on the $\mathrm{Ni}^{2+}$ core were increaged by one init, rithout changing the valenco-electron configuration, thexe would still be a ground state and various excited' states of the valence shell in this new system. Iel us now focus on just two states: the ground state " $\mathrm{g}$ ", and an excited (valence) gtate "e", of the same symmetry. The XPS shakerup spectra of transition-metal salts are usually explained in terms of a ground state configuration and a ligand-to-metal chargetransfex configuration, which would be ovx $g$ and $e$ - Referring to Fig. 7, we note that the valence configurations of states $g$ and $e$ (denoted by $\Psi_{g}$ and $\Psi_{e}$ ) will be esisentializy the same regardless of which core jelectron is missing. Thus for a Nika transition, $\Psi_{1 \mathrm{~g}} \cong \Psi_{2 g}$ and $\Psi_{1 e} \cong \Psi_{2 e}$, where 1 and 2 denote the 1 s and $2 p$ hole-state manifolds, respectively. Furthermore $\left(\Psi_{i e} \mid \Psi_{i g}\right)=0$ for $i=1,2$ of necessity. Thus $\left\langle\Psi_{i e} \mid \Psi_{j g}\right\rangle \geq 0$, where $i \neq j$. Now considex first $x P S$, then 
XES, sâillites.

In the Ni(2p) XPS spectrum, both the primary $2 p$ state $\left(P_{2}\right)$ and the secondary state $s_{2}$ will be populated. This follows because the overlap of $\Psi_{2 g}$ with the $\mathrm{NiF}_{6}^{4-}$ ground-state valenceielectron configuration will be substantially less than unity as a result of polaxitation toward the $\mathrm{Ni}^{3+}$ core. Much of this overiap is apparently taken up by the $\Psi_{z e}$ configuration, producing large XPS satellites. The same behavior would be expected for the $\mathrm{NiF}_{2}$ ls XPs spectrum, according to this picture:

Depopulations of the 1s-hole states would proceed almost entirely through channels $A$ and $D$, in preference to channels $B$ and $C$, because of the orthogonality of the $g$ and $E$ states. Insofar as the e-g splitting is nearly identical for the is and 2p manifolds, transitions $A$ and $\mathrm{D}$ would be nearly degenerate in energy, and only one peak would be observed in the XFS spectrum. Thus satellites would be intense in XFS but not apparent in XES. 


$$
-14-
$$

\section{REFERENCES}

This work was done with gupport from the U.s. Enezgy Research and pevelopment Administration.

1. CONDON E.V. and SHORTLEY G.K., "The Theory of Atomic spectra", (Cambridge University Fress, 1935); Chapter XV.

2. COMNERADE J.D., TRACY D., HAHSFIEID M.H.R., and THIMA $\mathrm{K}$., in "Vacuum Ultravlolet Radiation Physicg", edited by KOCH E.E., BAENSEI R., and KUNZ C. (Pergamon Vieweg, Braunsohweig, 1974), p. 243 .

3. BEFlaM B. and BUCHER A., Int. J. Mass. Spectrom. Ton Phys. 15 (3974) 463; BREHM B. and HÖFLER K., Ibid 17 (1975) 371.

4. HOTOP H, and MRHR D., J. Phy's. BB (1975) L.301.

5. LEE S.-T., SÜZER S., MRTHAIAS E., ROSENBERG R.A., and SHIRLEY U.A., J. Chent. Phys. 66 (1977) 2496.

6. KRAUSE M.0., CARLSON T.A., and DISHUKES R.D., Phys. Rev. 170 (1968) 37.

7. CHANG T.N, and POE, R.T., PhYs, Rev, Al2 (1975) 1432.

B. KRAUSE H.O., in proceedings of the International conference on the Physics of x-ray spectra, edited by DESIATMES R.D. (National Bureau of stándards, 1976).

9. MARTIN R.L. and SHIREEY D.A., PhYs. Rev. Al3 (1976) 1475.

10. KOHALCZYK S.P., LEY L., POLIAK R.A., MC FEELY F.R., and SHIRLEY D.A., Phys, Rev. B] (1973) 4009.

1i. Bagus P.S., FREEMAN A.J., and SASAKI F., Phys. Rev. Letters 30 (1973) 849. 
12. KOWALCZYK S.P., LEY L,, MC FEELY F.R., and SHIRLEY D.A., Phys. Rev. B11 (1975) 1721 .

13. FADLEY C.S., SHIRLEY D.A., FRGEMAN A.J., BAGUS, and MATLOW J.V., Phys. Rev. Letters 23 (1969) 1397.

14. BAgUS P.S., Phys: Rev. 139 (1965) A619.

15. LUYKEN, B.F.J., Physica 60 (1972) 432; LUYKEN B.F.J., DE HEER F.J., BAAS R.Ch., Physica 61 (1972) 200.

16. SPEARS D.P., FISCHBECK H.J., and CAFLSON T.A., PhYs. Rev. A9 (1974) 1603 .

17. COOPER J.H. and IA VILIA R.E., Phys. Rev. Letters 25 (1970) 1745.

18. MARTIN R.L., KOWLLCZYK S.P., and SHIRLEY D.A., Lancence Berkeley Laboratory Report LAL-5445 (1977), submitted to J. Chem. Phys.

19. AMUSIA M.Ya., IVANOV V.K., CHEREPKOV N.A., and CHERNYSHEVA I.V., Phys, Iett. A40 (1972) 361 .

20. ADAM M.Y., WUILLEUMIER F., SANDERS N., SCHMIDT V., MEHL.HORN W., and WENDIN G., Paper I-27, This Conference.

21. WENDIN G. and OHNO M., Physica Scripta 14 (1976) 148.

22. LUKIRSKII A.P., ZIKKINA T.M., and BRITOV :.A., Akad. Nauk. SSSR, Sex. F12 28 (1964) 7h2, CODLING $K$. and MADDEN R.P., Appl. Opt. 4 (196.5) 1431; HAENSEL R.. KEITEL G., SCHREIBER P., and KUNZ C., Phys. Rev. 188 (1969) 1375.

23. GELIUS U., J. Electr. Spectr. 5 (1974) 985.

24. MC GUIRE E.J., Phys. Rev. A9 (1974) 1840.

25. SÜZER S., IEE S.-T., and SHIRLEY D.A., PhYS. Rev. AIJ (1976) 1842.

26. SÜzER S. and SHIRLEY D.A., J. Chem. PhYs. 6l (1974) 2481. 


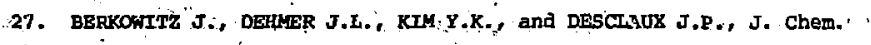
Phys. 61 (1974) 2556 .

28. KIM Y.Y. and BAgus P.S., Phys. Rev: AS (1973). 2739, also HANSEN J.E., private communication to SHIRLEY D.A. (1976). ,

29. SÜZER S.. BANNA M.S.., and SHIRLEY D.A., J. Chiem. Fhys. 53 (1975) 3473 .

30. SÜZER S., LFE S.-T., and SHIRLEY D.A., J. Chem. FhYs. 65 (1976) 412...

31. Reference 1, Chapter 11.

32. ASADA S., SATOKO C., and SUgano S., J. PhYs. Soc, (Japan) 37 (1975) 8555 . 
TABLE I. ARGON XPS AND XES SATFLIITE INTENSITIES ${ }^{a}$

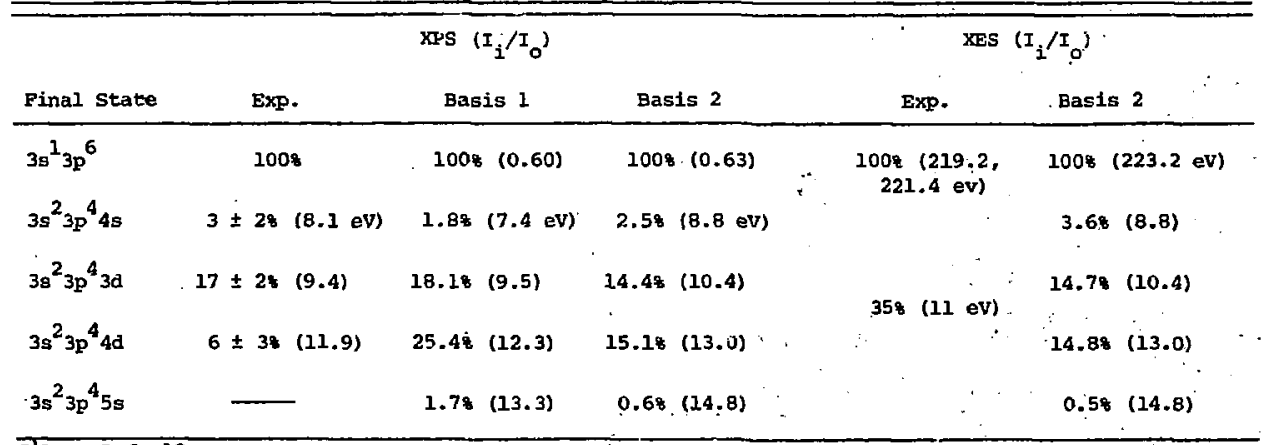

a) From Ref. 18 . 
Y

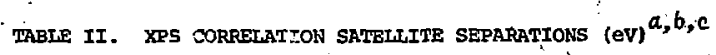

\begin{tabular}{|c|c|c|c|c|c|}
\hline $\mathrm{nl}=3 \mathrm{~s}$ & $\left(\mathrm{Cl}^{-}\right)_{6}$ & (Ar) & $11(258)$ & $\left(\mathrm{K}^{+}\right) 14$ & $\left(\mathrm{Ca}^{+2}\right) 16$ \\
\hline 45 & $\left(\mathrm{Bx}^{-}\right)^{4}$ & $(\mathrm{Kr})$ & $6.5(30 \%)$ & $\left(\mathrm{Rb}^{+}\right) 11$ & $\left(\mathrm{sr}^{+2}\right) 13: 5$ \\
\hline 58 & (I') 3.6 & $(\mathrm{Xe})$ & $6.0(58 \%)$ & $\left(\mathrm{Cs}^{+}\right) 9: 3$ & $\left(\mathrm{Ba}^{+2}\right) \cdot 9.9$ \\
\hline
\end{tabular}

a) From Ref. 18. with the exception of the raxe gases, the entries in this table. are taken from XPS measurements of appropriate salts. In those cases where a given ion has been studied in several salts (e.g., $\mathrm{K}^{+}$. in $\mathrm{KF}, \mathrm{KCl}$, and. $\mathrm{KBr}$ ), the satellite separation is an average of the observed separations. In most instarices; the deviations from salt to salt are within the experimental uncertninty in the separations ( $(.2, \mathrm{eV})$.

b) Parenthetical entries for the rare gases are the relative intensity of the. correlation satellite to the primary peak.

: c) S. P. Kowalczyk, "Photoelectron Spectroscopy and Auger Electron spectroscopy of Solids and Surfaces", Ph.D. Thesis, Lawxence Bexkeley Laboratory Report IBL-4319 (1976), p. 131 .. 
FIGURE CAPTIONS

Fig. 1 Levels in Ba and $\mathrm{Ba}^{+}$excited by freIa radiation (left) and the photoelectron spectrum fxom this syatem (right).

Fig. 2 Dlagram illustrating FISCI accompanying 18 photoemiaston in nean. After Chang and Poe (Ref. 7). The process depicted is 1s photoionization, to the $2 \mathrm{~s} 2 \mathrm{~s}^{2} 2 \mathrm{p}^{5} 3 \mathrm{p}$ state in $\mathrm{Ne}^{+}$.

Fig. 3 Diagram illustrating FISCI accompanyting 3s photoemission from ${ }^{+}$, Ar, other $3 \mathrm{~s}^{2} 3 \mathrm{p}^{6} \ldots$ cases, or ns photoemission from $n s^{2}{ }^{\prime}{ }^{6}$ cases.

Fig. 4 Diagram of FIsCI processes in $\mathrm{Xe}^{+}(4 \mathrm{p})$ photoemission. (a) virtual super Coster-Kronig processes, after Wendin and Ohno, Ref. 21. (b) a contributing diagram in which the $4 d^{8} 4 f$ final state is speciflcally recognized, :0 emphasize the similarity to the 3s-hole core of Fig. 3 .

Fig. 5 The ISCI process $4 p^{2}+4 p$, admixel into the initial state of Ca.

Fig. 6 The $\mathrm{Ca}^{+}$(Her) spectrum, showing the $4 s, 3 d, 4 \mathrm{p}, 5 \mathrm{~s}$, and $4 d$ final states, right to left.

Flg. 7 Level dlasxam for core-tomore x-ray transition. Channels A and $D$ are most intense and close in energy, as explalied in text, precluding Intense satellites. 




Fig. 1 


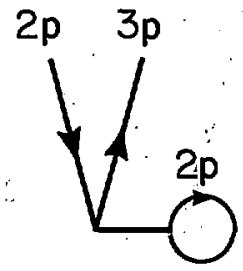

is $\in p$

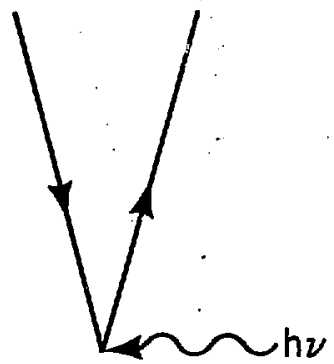

Fig. 2

XBL 778-1770A 
$.22-$

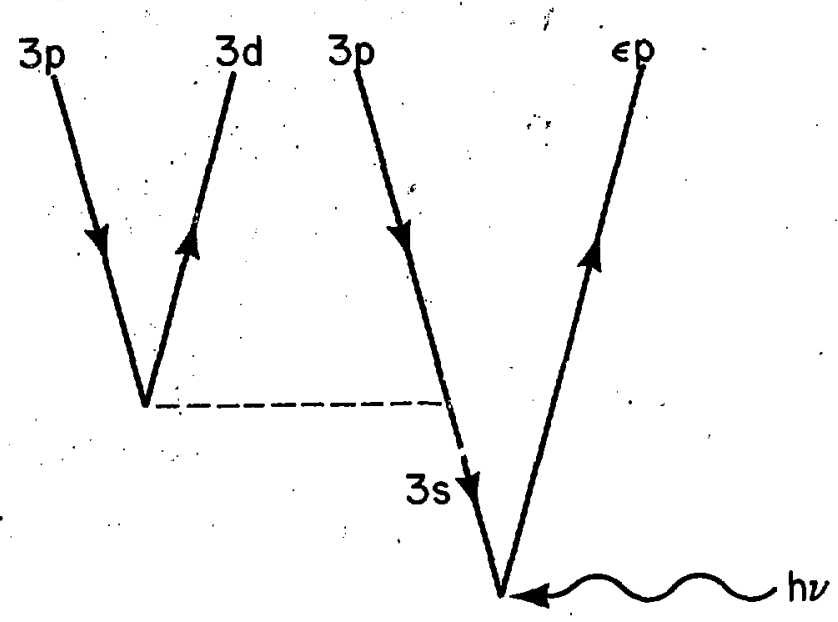

Fig. 3

XBL $778-17698$ 

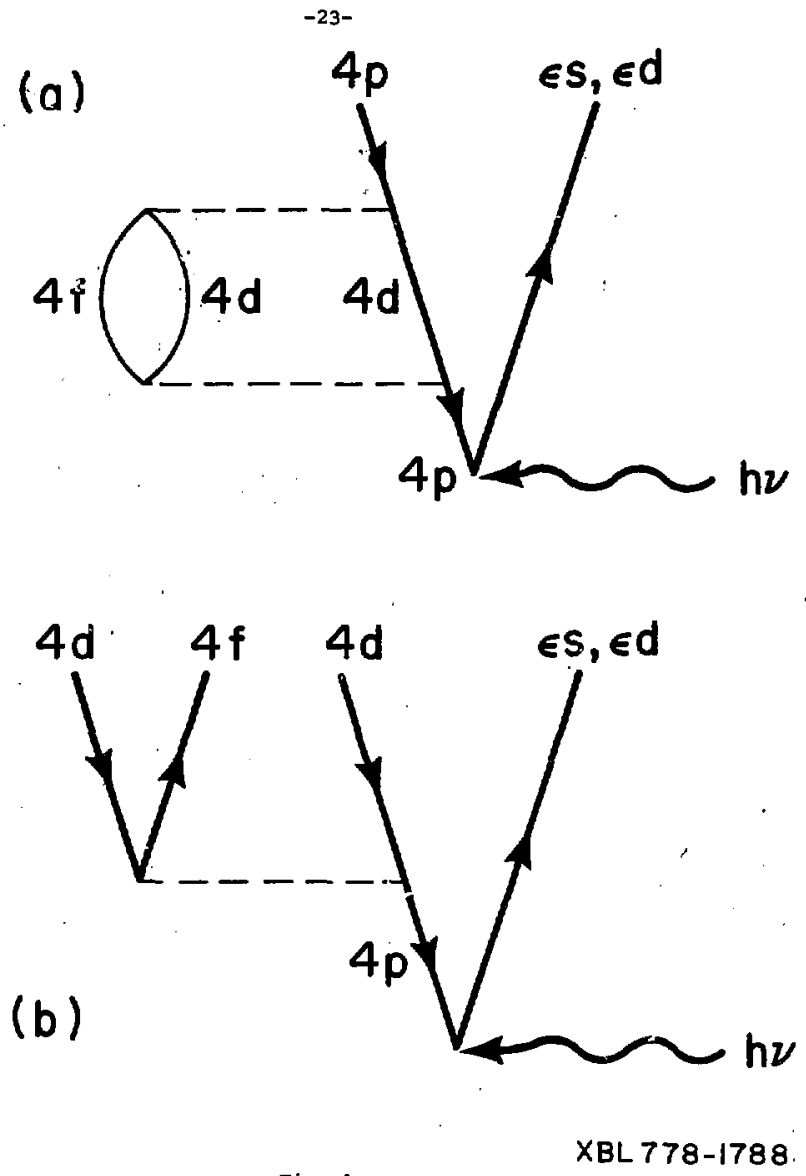

Fig. 4 


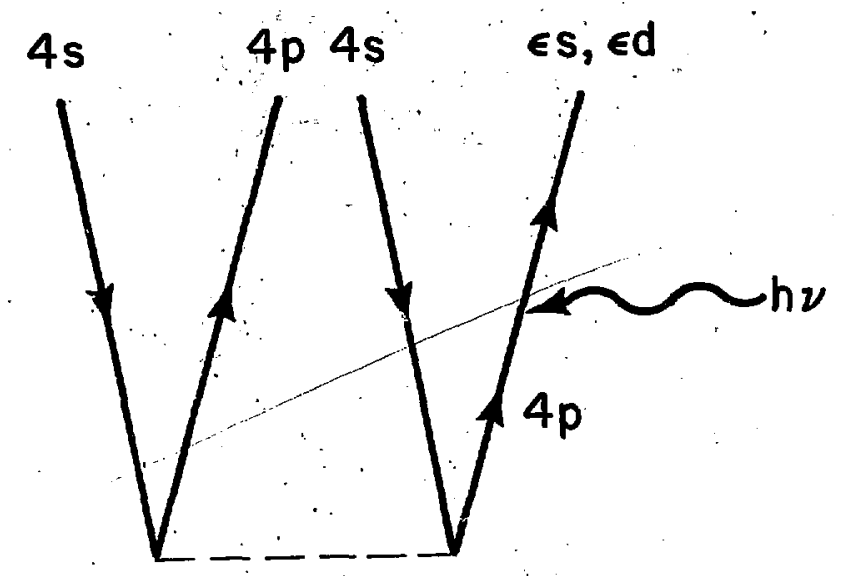

XBL 778-1789 


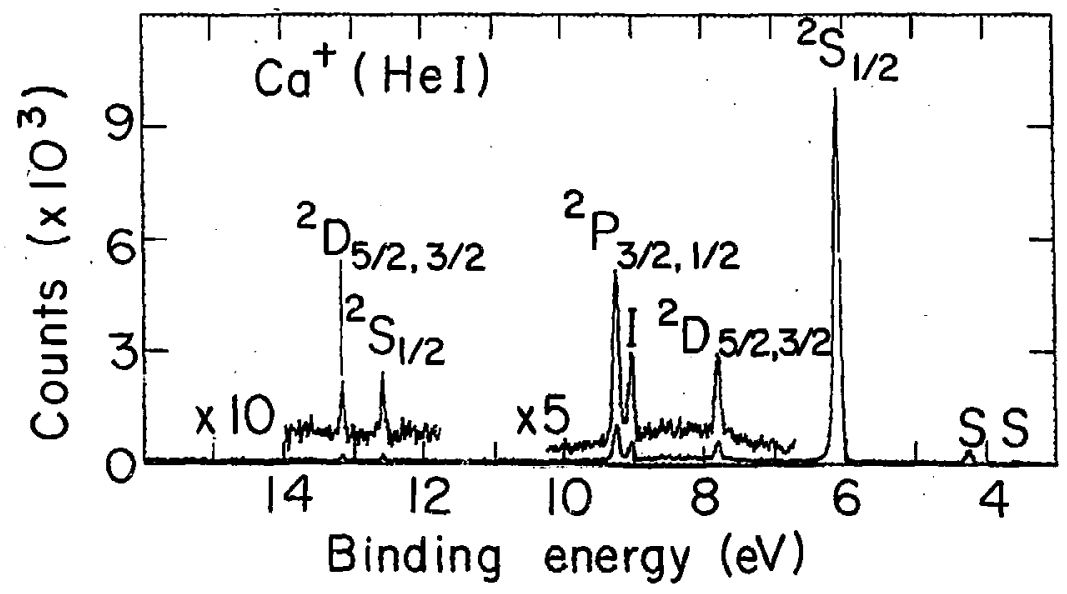




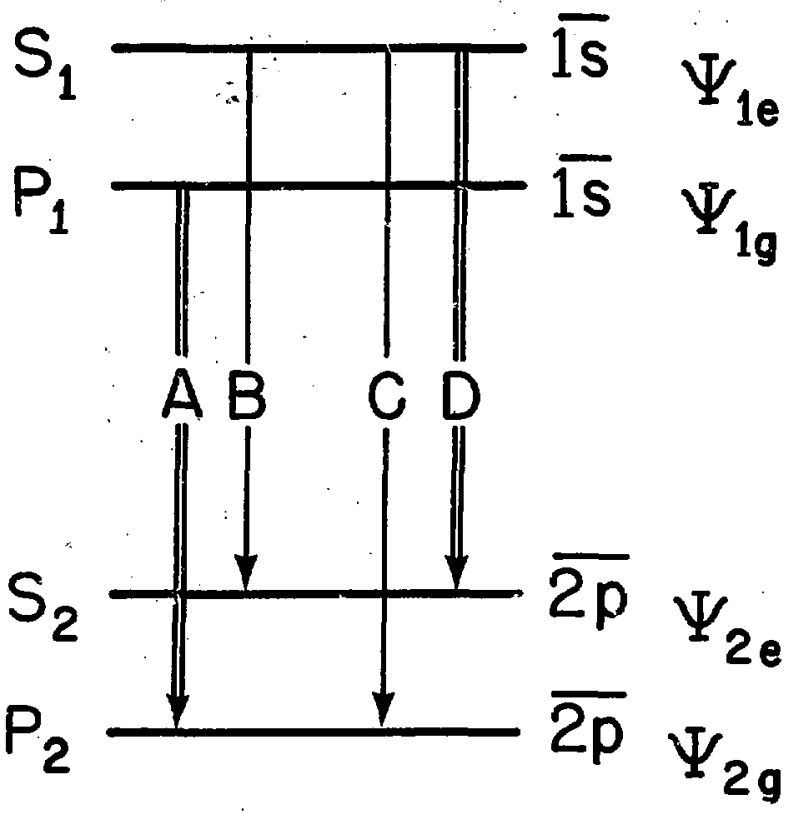

Fig. 7

XBL 778-1772 